\title{
Adaptive Non-Cartesian Networks for Vision
}

\author{
$\dagger, \uparrow \dagger J . R$. Serra and $\dagger$ J. Brian Subirana \\ $\dagger$ Dipartimento di Sistemi e Informatica \\ Università degli Studi di Firenze \\ Via S. Marta 3, 50139 Firenze, Italy \\ †DDepartamento de Informática. Edificio C \\ Universidad Autónoma de Barcelona. \\ 08193 Barcelona, Spain \\ e-mail: joanr@aguirre.ing.unifi.it, brian@upisun1.uab.es
}

\begin{abstract}
We address the problem of locating and extracting frame curves on interesting image areas. Reference frames, focus of attention, bounding contours of shapes, axis of inertia, centers of masses and other mid-level visual structures, can be used to guide other mid-level visual tasks or to lead subsequent high level processing like recognition, indexing or image retrieval. Frame curves, are useful to tackle non-rigid object recognition problems because these have fuzzy boundaries. Where is $t$ he boundary of a cloud, oak leave or a leopard? We present a perceptual organization approach based on dynamic programming and adaptive noncartesian networks, a new kind of networks which are based on placing processor lines using a distribution function adapted to the image array. We present a novel computational framework to extract frame curves directly on the image and several experiments on real images.
\end{abstract}

\section{Introduction}

Recent work in computer vision is based on the assumption that edge detection precedes grouping and object recognition. Most existing approaches, are based on an edge detection stage, a example of such approach is [Havaldar et al. 1994]. An edge detection stage, implies an information loss (the edges of an image do not include all the features). After the edge detection stage, such schemes make an edge linking task to obtain perceptual groups. We call these methods, indirect methods.

We (like others) argue against the assumption that edge detection stage precedes grouping and object recognition and suggest that grouping precedes the computation of discontinuities and most other early visual tasks.

Some structures like the axis of inertia and bounding shape contours are not defined locally neither in the image nor in the scene. We cannot find them by process values near particular image locations (like many early vision tasks do).

The computational problem that we are interested in is that of finding curves and points (that we call frame curves) in images that have certain properties that are not defined locally and investigate robust and direct perceptual organization schemes that can compute frame curves, without relying on existing edge 
detectors. Other approaches such as Active Contour models (snake-based), see [Amini, Tehrani and Weymouth 1988], need an important initialization stage. The snakes must be near the structures.

We argue that approaches based on cartesian and non-cartesian random networks (see [Subirana-Vilanova 1993] and [Serra and Subirana 1996]), are not optimal for vision. There are two reasons: the first one is that they lack accuracy to locate image structures, and the second is that they don't estimate their curvature closely.

Here we present a novel two stage approach: the first one is filter based whereas the second one consists on frame curve extraction. It is made through a new kind of random networks, adaptive non-cartesian networks $(\mathcal{A N C N})$. These networks are able to locate closely image structures and estimate their curvature correctly. First, we compute the feature inertia surfaces $(\mathcal{F} \mathcal{I S})$, after this, we calculate, for a particular image salient structure $\mathcal{E}$, its distribution function of orientations $\mathcal{D}^{\mathcal{E}}$. With this function, we generate after an $\mathcal{A N C N}$. With a dynamic programming approach, in the second step we extract the frame curves for the different structures of type $\mathcal{E}$ present on the image. Previous approaches on adaptive processing such as the surface reconstruction methods introduced in [Vasilescu and Terzopoulos 1992], try to match surfaces as an input. In these cases the networks are called meshes.

\section{Adaptive non-cartesian networks}

Suppose that we want to find different structures of type $\mathcal{E}$, on an image $\mathcal{I}_{n x n}$ as input, by the extraction of a set of frame curves. To obtain this goal, first we should locate them: where are frame curves located on $\mathcal{I}_{n, x}$ ? Second, after locating them, we should construct a search space of curves for frame curve extraction. In this section our goal is locate "likely points for", or "relevant" image salient structures of type $\mathcal{E}$, and obtain a data structure to guide the generation of the search space (network). We should locate salient areas such that frame curves cross them. To locate these areas, we propose an oriented filters based approach. Suppose that we have a filter $\mathcal{F} \mathcal{E}$, that gives high responses on areas where there are $\mathcal{E}$ structures. We call these high likelihood salient areas the feature inertia surfaces because frame curves will cross them like curved axis of inertia. These surfaces point out regions that frame curves occupy on the image. We can express them as follows, for a given point $\mathbf{p}$ and an orientation $\theta_{i}$ :

$$
\mathcal{F I}_{i}^{\mathcal{E}}(\mathbf{p})=\left(\mathcal{F}^{\mathcal{E}}\left(\theta_{i}\right) * \mathcal{I}\right)(\mathbf{p})
$$

The points with high response, are the salient points, and its associated orientation is the orientation of the particular filter. To guide an efficient generation of the search space of curves, we construct the set of salient points with the associated orientation. It can be expressed as follows:

$$
\mathcal{P}_{\text {oints }}\left(\theta_{i}\right)=\left\{\mathbf{p} \mid \mathcal{F} \mathcal{I} \mathcal{S}_{i}^{\mathcal{E}}(\mathbf{p}) \geq r_{0}\right\}
$$


Where $r_{0}$, is the minimum response. If the response for a given point is greater than $r_{0}$, this point belongs to the set. As an example of these, see figure 1 . In this case we suppose that we are looking for perceptual groups on brightness, we have chosen a filter that produces high responses near object locations, this filter is the $\mathcal{D O O G} 2$ filter of [Young 1985].

When feature inertia surfaces have been located, we need to define a search space of curves and extract from them the frame curves. A network, will be formed by a set of lines from side to side of an image. A curve over a network is a set of consecutive line segments. Which is the minimum network, (minimum number of lines) to find the shape of a leopard? Cartesian networks (see [Sha'ashua and Ullman 1988]), have an elevate number of lines and have an orientation dependency such that certain orientations are favored. This means that some curves extracted from it will not be good approximations to image structures, due the problem of estimating some curvature that cartesian networks has. To solve this problem, in [SubiranaVilanova 1993], another type of network was introduced, non-cartesian random networks. In this kind of networks, the line slopes follows a uniform distribution. If we define a line like a: $y \cos \alpha-x \sin \alpha+\rho=0$ then, $\alpha \in \mathcal{U}[0, \pi]$. In this case, no orientation is favored, but original cartesian and non-cartesian random networks have another problem: on both cases, lines are uniformly distributed over the image, this implies the assumption that salient structures will be equally distributed on the image plane. This is generally false because the probability to locate one structure on a certain image area is not the same for all image locations. For example, group elements are located near object boundaries and on these two types of networks information like this is not used. This type of information is relevant to decide where we must concentrate network processing.

Then non-cartesian random networks do not provide accuracy to locate the

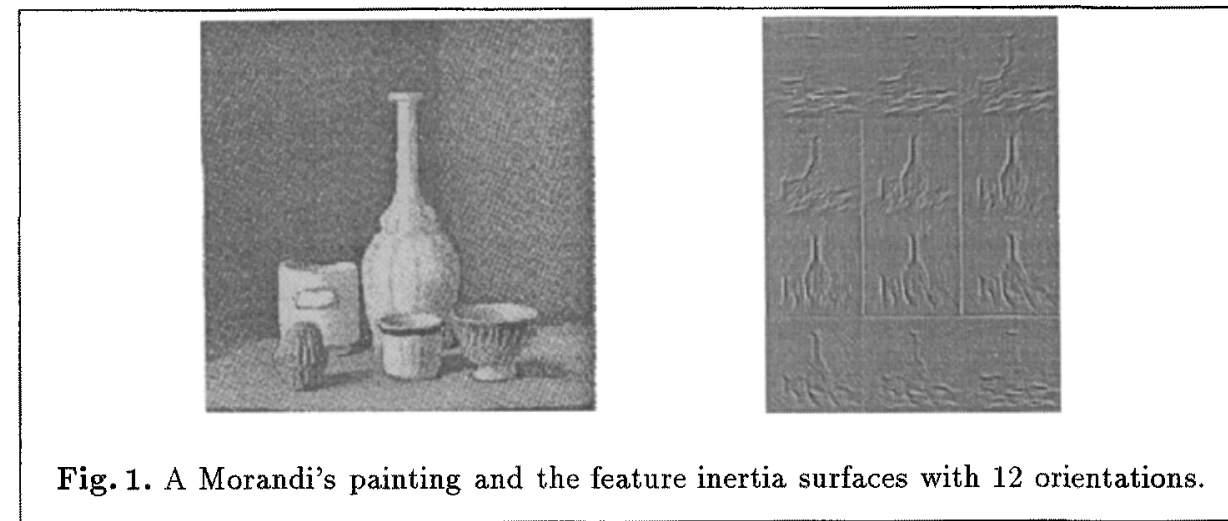

structures. Why uniform distribution of slopes? Which is the best distribution of slopes to recognize a leopard? Adaptive non-cartesian networks $(\mathcal{A N C N})$, a new kind of networks address some of the problems mentioned above. These networks locate salient structures since their generation is guided by the feature inertia surfaces and the points set. 


\section{Frame curve extraction}

\section{Adaptive non-cartesian network generation}

An $\mathcal{A N C N}$ defines a search space of curves. For a given type of structure $\mathcal{E}$ and its feature inertia surfaces $\mathcal{F I} \mathcal{S}_{i}^{\mathcal{E}}$, we define the inertia of a curve $\mathcal{I C} \mathcal{C}^{\mathcal{E}}$. Long and smooth curves will be the most salient, like on the [Sha'ashua and Ullman 1988] model. A network will be formed by a fixed set of lines, these lines will be located near the $\mathcal{E}$ structures present on the image. From the feature inertia surfaces that embodies information about structure locations, we will construct an adaptive non-cartesian network. An important issue is: what number of orientations should our networks have? We will estimate the probability distribution function of orientations $\mathcal{D}^{\mathcal{E}}$, to know which are the most important lines. This means that, for each orientation $\theta_{i}$ used on a filtering step we calculate the probability that an oriented filter $\mathcal{F}^{\mathcal{E}}\left(\theta_{i}\right)$ will give us high responses. With this approach, we generate networks of $n$ lines and choose the most important ones. In terms of probabilities, this is to choose $n$ lines such that their orientations have high probability by the function $\mathcal{D}^{\mathcal{E}}$. To achieve this goal, we should begin to see what is the probability density function of orientations $d e^{\mathcal{E}}$, and after we should deduce the probability distribution function of orientations $\mathcal{D}^{\mathcal{E}}$. The probability density function $d e^{\mathcal{E}}$, for an image $I_{n, x n}$ and an orientation $\theta_{i}$ is:

$$
d e^{\mathcal{E}}\left(\theta_{i}\right)=\frac{\# \mathcal{P}_{\text {oints }}\left(\theta_{i}\right)}{n^{2}}
$$

Where \#P्ints $\left(\theta_{i}\right)$, is the number of elements in $\mathcal{P}$ oints $\left(\theta_{i}\right)$. Using this function, to obtain the distribution function we should do the following accumulated operation:

$$
\begin{aligned}
& \mathcal{D}^{\mathcal{E}}\left(\theta_{0}\right)=d e^{\mathcal{E}}\left(\theta_{0}\right) \\
& \mathcal{D}^{\mathcal{E}}\left(\theta_{i}\right)=\mathcal{D}^{\mathcal{E}}\left(\theta_{i-1}\right)+d e^{\mathcal{E}}\left(\theta_{i}\right)
\end{aligned}
$$

How can we generate an adaptive non-cartesian network from function $\mathcal{D}^{\varepsilon}$ ? One elegant solution is to construct a random number generator $(\mathcal{G})$, that follows the distribution $\mathcal{D}^{\mathcal{E}}$. To do this, we need two steps:

$$
\begin{aligned}
& \mathcal{X}=\max \left(\mathcal{D}^{\mathcal{E}}\right) \\
& \mathcal{G}\left(\mathcal{D}^{\mathcal{E}}\right)=\left(\mathcal{D}^{\mathcal{E}}\right)^{-1}\left(x_{i}\right), \quad x_{i} \in \mathcal{U}[0, \mathcal{X}]
\end{aligned}
$$

To generate an adaptive network with $l$ lines, we should generate $l$ random numbers uniformly distributed on the interval 0 and the maximum of the distribution function $\mathcal{D}^{\mathcal{E}}$. For each one, we should do the inverse image of the function $\mathcal{D}^{\mathcal{E}}$. This gives us an orientation $\theta_{k}$, now we choose a point $p$ such that $p \in \mathcal{P}_{\text {oints }}\left(\theta_{k}\right)$, to generate the lines.

If there are noisy areas in the image, the filters give high responses on $t$ hem. In order to avoid line accumulation in, we impose that to become a valid candidate line for a network, the inertia $\mathcal{I}^{\mathcal{E}}$, must be higher than $i_{0}$. Where $\mathcal{I}^{\mathcal{E}}$ is:

Definition: Inertia of a line $\mathcal{I}^{\mathcal{E}}$. A line defined by the equation: $y \cos \theta_{i}-x \sin \theta_{i}+$ $\rho_{k}=0$ where $L_{k}^{\theta_{i}}(x, y)$ is the set of points such that $y \cos \theta_{i}-x \sin \theta_{i}+\rho_{k}=0$, has an inertia defined by: 


$$
\mathcal{I}^{\mathcal{E}}\left(L_{k}^{\theta_{z}}\right)=\int^{L_{k}^{\theta_{z}}} \mathcal{F} \mathcal{I} \mathcal{S}_{i}^{\mathcal{E}}(u) d u
$$

Where the function $\mathcal{F I} \mathcal{S}_{i}^{\mathcal{E}}$, is the feature inertia surface for the orientation $\theta_{i}$. If the inertia of a line is lower than the minimum inertia $i_{0}$, then this line can not be a component of the network, this is, if $\mathcal{I}^{\mathcal{E}}\left(L_{k}^{\theta_{v}}\right)<i_{0}$, the line $L_{k}^{\theta_{i}}$ does not belong to the network. The number of lines on a network, will be a function of the resolution we are willing to reach. We choose here to generate all the available lines, this is, to generate lines until the set $\mathcal{P}$ oints becomes empty. In this case the generation process does not need an extra parameter for the number lines.

\section{Dynamic computing and frame curve extraction}

When we have generated the network, we have a search space of curves. These curves match different structures present in the image. Through the next calculation, we obtain the inertia $\left(\mathcal{I C} \mathcal{C}^{\mathcal{E}}\right)$ for the curves present over the network. The most important ones will be frame curves for the different $\mathcal{E}$ structures present in the image. For each curve $\mathcal{C}$ over the network, we compute:

$$
\mathcal{I} \mathcal{C}^{\mathcal{E}}(\mathcal{C})=\int^{\mathcal{C}} \mathcal{F} \mathcal{I} \mathcal{S}_{\theta(u)}^{\mathcal{E}}(u) \rho \int_{0}^{u} \frac{1}{\alpha \mathcal{T}(t)} d t d u
$$

Where $\theta(u)$ gives us the associated orientation of the point $u$. $\mathcal{T} l, \rho$, and $\alpha$ are the penetration factor, circle constant, and the tolerated length respectively. A complete description of the meaning of these parameters can be seen in [SubiranaVilanova 1993]. $\mathcal{T} l$ is a function of the curvature connection. To realize this computation, we use a dynamic programming approach. For each pixel pe oriented $\theta_{i}$ radians in the network, for all its connections $k$ we make this iterative computation:

$$
\begin{aligned}
& \mathcal{G C I}^{(0)}\left(\mathbf{p e}_{\mathbf{e}}\right)=\mathcal{F I}_{i}^{\mathcal{E}}\left(\mathbf{p}_{\mathbf{e}}\right) \\
& \mathcal{G C I}^{(n)}\left(\mathbf{p}_{\mathbf{e}}\right)=\max _{k}\left\{\mathcal{F I} \mathcal{S}_{i}^{\mathcal{E}}\left(\mathbf{p e}_{\mathbf{e}}\right)+\mathcal{G C I}_{k}^{(n-1)}\left(\mathbf{p e}_{\mathbf{e}}\right) \rho^{1 / \alpha \mathcal{T} l}\right\}
\end{aligned}
$$

Where $\mathcal{G C I}^{(n)}$ (pe) (Global Curve Inertia), points out the inertia of the best curve of length $n$ that begins at pe.

We need to do a number of iterations function of the total length of the curve that we will need to extract. Formally, it is needed to do a number of iterations such that the series 6 reaches the convergence as point out [Alter and Basri 1995]. It is sufficient to do a number of iterations equal to the number of the longest curve that we want to search. Indeed, in [Sha'ashua and Ullman 1988], the authors claim that "a little number of iterations" in most cases is sufficient. If we are searching curves of 100 pixels of length, the method sure us that with 100 iterations, we will obtain the best curve of length 100. But in general, which is the number of iterations? We should fix it in each execution, for example, it may be $x$ times the size of the image. 


\section{Perceptual organization}

Our scheme is designed to work for brightness, texture and color but our implementation deals only with brightness. Perceptual organization process can be seen as an instance of finding salient structures where $\mathcal{E}$ are perceptual groups. The frame curves for groups will be closed curves with the same initial and final points. In this section, we present an implementation for grouping on brightnes$s$, but it is easy to extend these ideas to other kinds of images, such as color or texture, see [Serra and Subirana 1996]. In the brightness case, our adaptive networks should concentrate its lines in the nearness of object boundaries. For this reason, we should choose a filter that has the property of giving high responses near object boundaries. The $\mathcal{D O O} \mathcal{O} 2$ filter is an oriented filter defined in [Young 1985].
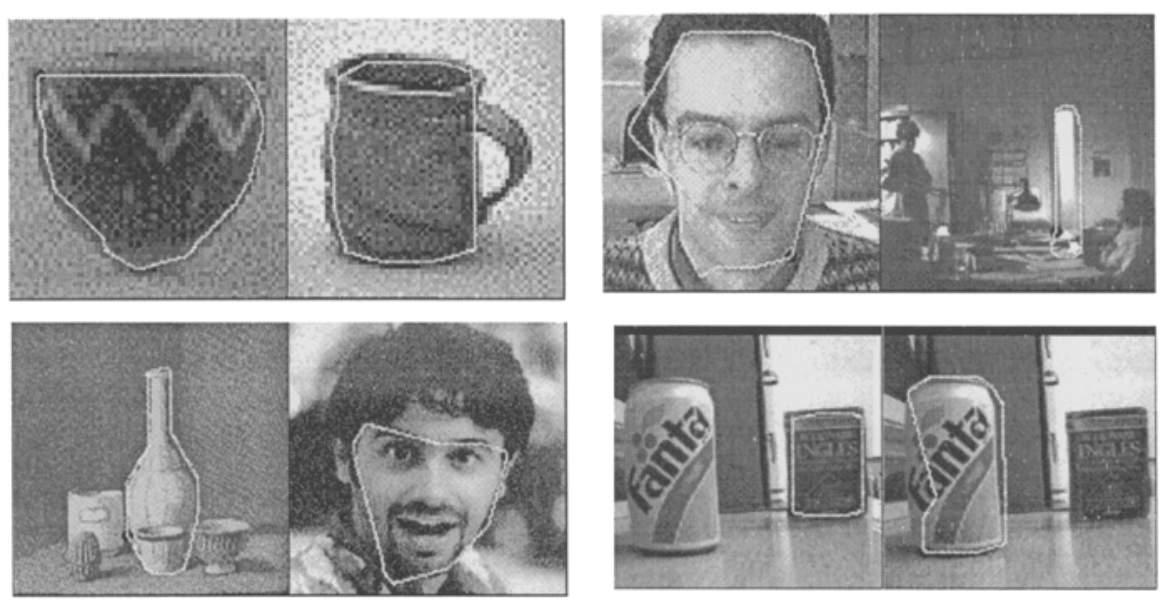

Fig. 2. Top left: the size of the network is 227 lines, we have done 128 iterations and the length of the frame curve resultant is 327 pixels. Top second: the size is 128 lines, and we have done 256 iterations and the length of the frame curve is 292 pixels. Top third: the size of the network is 250 lines, we have done 128 iterations and the length of the frame curve resultant is 337 pixels. Top right: the size is 217 lines, and we have done 128 iterations and the length of the frame curve is 160 pixels. Bottom left: the size of the network is 175 lines, we have done 256 iterations and the length of the frame curve resultant is 226 pixels. Bottom second: the size is 257 lines, and we have done 256 iterations and the length of the frame curve is 239. Bottom third and bottom right the size of network is 206 lines, we have do 128 iterations and the length of the frame curves recovered is 156 and 242 pixels repectively .

Using $\mathcal{D O} \mathcal{O} \mathcal{G} 2$ filter, we obtain the feature inertia surfaces to do a grouping process. The filtering step produces, in the nearness of object boundaries, what we call bundle of lines. 
Definition: Bundle of lines of wide $n$. It is formed when, by the filtering, a feature inertia area has a wide bigger than $n$ pixels. If for a given orientation $\theta_{i}$, then exist two values $k_{1}, k_{2}$ such that:

$$
d\left(L_{k_{1}}^{\theta_{i}}, L_{k_{2}}^{\theta_{i}}\right)<n
$$

When we generate an adaptive non-cartesian network, we choose only one line representing this bundle. This line is the most important one, this is, $L_{k}^{\theta_{i}}$ such that:

$$
\mathcal{I}^{\mathcal{E}}\left(L_{k}^{\theta_{2}}\right)=\max _{k_{1}}\left\{\mathcal{I}^{\mathcal{E}}\left(L_{k_{1}}^{\theta_{i}}\right)\right\}
$$

A bundle of lines imply that more lines than the needed ones will be generated on the network generation step. For this reason, we will choose only one line of these bundles, this will be the most important line, following the definition of the last section. In the implementation (over a Pentium 200Mhz with $32 \mathrm{Mbytes}$ of RAM), we have taken 12 orientations, $r_{0}=160, \mathcal{T} l=r_{c}$, this is the radius of curvature of the connection, $a=-1$ and $\rho=e$. In the filter, it has taken $\sigma=r=2$. On the networks, we have considered a maximum of 4 connections per pixel, two for both sides of a pixel, besides the connection that the line support gives per pixel. The resolution of the networks is $\frac{1}{2}$ the image size, in all cases $256 x 256$ pixels. The number of lines, is chosen automatically, as we have explained on the last section. In the figure 2, we can see a set of experiments on real images. In all these figures, the black point over the frame curve points out its initial and final points (the total time of computation is more or less 3 minutes for one image).

To extract multiple relevant structures, not only the first one, we should consider these points:

- Will it always be possible? As pointed out in [Alter and Basri 1995], on cluttered images it will not be possible to extract all the structures with a network. In some cases, the frame curves extracted joint two or more structures. One should remember that the network chooses only one connection per pixel as a result for a curve. On tidy images, where different structures are separated we can do a full correct extraction.

- How can we proceed? When we have extracted the first structure, we should do, for the frame curve, the next computation: for each pixel pe of the frame curve, $\mathcal{G C I}(\mathbf{p e})=0$. And finally, we can go on extracting frame curves to recover subsequent structures. Future research is needed here to study different ways to do this computation. In the figure 2 (bottom third and bottom right) we can see an example.

\section{$5 \quad$ Summary and Conclusions}

The most novel contribution of our work is that it uses adaptive non-cartesian networks. These networks improve the non-cartesian random networks of [SubiranaVilanova 1993] and [Serra and Subirana 1996]. Like in their scheme, 
does not have a small and fixed subset of orientations but instead of using a uniform distribution, we use a probability distribution that adapts itself to the image characteristics. All orientations are equally considered to be represented, unlike in a cartesian network, where only vertical, horizontal and diagonal connections are considered. Adaptive non-cartesian networks are also interesting because processing can be selectively targeted at certain regions of the image array. This is different from previous approaches which use cartesian networks and therefore have the same density of processors in all areas of the image array. Our scheme allows an efficient and accurate extraction of different kinds of salient structures. With a small number of lines we improve the convergence time and the memory space required. We have shown the application to the perceptual grouping process and have introduced the notion of network exploration for subsequent processing. Our scheme computes global structures directly on the image and merges early and mid-level vision on a framework that may run with several kinds of images, without the need of an early vision module such as edge-detection. In particular, our scheme may be used to integrate brightness, color and texture.

\section{Acknowledgements}

We acknowledge the help from Stefano Casadei from MIT LIDS and the interesting suggestions from Professor Alberto Del Bimbo, Jacopo Corridoni and Pietro Pala from Image Science and Technology Center, Florence University.

\section{References}

[Amini, Tehrani and Weymouth 1988] Amir A. Amini, Saeid Tehrani and Terry E. Weymouth. Using dynamic programming for minimizing the energy of active contours in the presence of hard constraints. In Proceedings of Second International Conference on Computer Vision, pages 95-99, Florida 1988.

[Alter and Basri 1995] T.D. Alter and R. Basri. Extracting salient curves from images: an analysis of the saliency network. MIT AI TR 1550, 1995.

[Havaldar et al. 1994] P. Havaldar, G. Medioni and F. Stein. Extraction of groups for recognition. In Proceedings of the European Conference on Computer Vision, pages 251-261, 1994

[Serra and Subirana 1996] J. R. Serra and J. B. Subirana. Perceptual Grouping on Texture Images using Non-Cartesian Networks. In Proceedings of 13th International Conference on Pattern Recognition. Vol. II, pages 462-466. Vienna, 1996.

[SubiranaVilanova 1993] J. B. SubiranaVilanova. Middle level vision and visual recognition of non-rigid objects. MIT PhD thesis. August 1993.

[Sha'ashua and Ullman 1988] A. Sha'ashua and S. Ullman. Structural saliency: The detection of globally salient structures using a locally connected network. Proceedings ICCV 2, pages 321-327, 1988.

[Vasilescu and Terzopoulos 1992] M. Vasilescu and D. Terzopoulos. Adaptive meshes and shells: irregular triangulation, discontinuities, and hierarchical subdivision. In Proceedings of IEEE International Conference on Computer Vision and Pattern Recognition, pages 829-831, Illinois 1992 .

[Young 1985] R. Young. The gaussian derivative theory of spatial vision: Analysis of cortical cell receptive field line-weighting profles. Technical Report GMR-4920. General Motors Research, 1985. 